\title{
Establishing a novel prediction model for improving the positive rate of prostate biopsy
}

\author{
Tao Tao ${ }^{1 \#}$, Deyun Shen ${ }^{1 \#}$, Lei Yuan ${ }^{2 \#}$, Ailiang Zeng ${ }^{3,4}$, Kaiguo Xia ${ }^{1},{\text { Bin } \text { Li }^{1} \text {, Qingyu Ge }}^{1}$, Jun Xiao ${ }^{1}$ \\ ${ }^{1}$ Department of Urology, ${ }^{2}$ Department of Radiology, The First Affiliated Hospital of USTC, Division of Life Sciences and Medicine, University of \\ Science and Technology of China, Hefei 230001, China; ${ }^{3}$ Department of Neurosurgery, The First Affiliated Hospital of Nanjing Medical University, \\ Nanjing 210006, China; ${ }^{4}$ Department of Neurology, Brigham and Women's Hospital and Harvard Medical School, Boston, MA, USA \\ Contributions: (I) Conception and design: J Xiao, T Tao; (II) Administrative support: J Xiao; (III) Provision of study materials or patients: J Xiao, T \\ Tao, D Shen, L Yuan; (IV) Collection and assembly of data: D Shen, K Xia, B Li, Q Ge; (V) Data analysis and interpretation: D Shen, A Zeng; (VI) \\ Manuscript writing: All authors; (VII) Final approval of manuscript: All authors. \\ \#These authors contributed equally to this work. \\ Correspondence to: Jun Xiao. Department of Urology, The First Affiliated Hospital of USTC, Division of Life Sciences and Medicine, University of \\ Science and Technology of China, Hefei 230001, China. Email: ustc_urology@126.com.
}

Background: At present, prostate-specific antigen (PSA) is the primary evaluation index for judging the necessity of prostate cancer (PCa) biopsy. However, there is a high false-positive rate and a low predictive value due to many interference factors. In this study, we tried to find a novel prediction model that could improve the positive rate of prostate biopsy and reduce unnecessary biopsy.

Methods: We retrospectively studied 237 patients, including their age, body mass index (BMI), PSA, prostate volume (PV), prostate imaging-reporting and data system (PI-RADS) v2 score, neutrophillymphocyte ratio (NLR), biopsy Gleason score (BGS), and other information. The univariate and multivariate logistic analyses were used to screen out indicators related to PCa. After establishing a prediction formula model, we used receiver operating characteristic (ROC) curves to assess its prediction performance.

Results: Our study found that age, PSA, PI-RADS v2 score, and diabetes significantly correlated with PCa. Based on multivariate logistic regression analysis results, we created the following prediction formula: $\mathrm{Y}=$ $2.599 \times$ PI-RADS v2 score $+1.766 \times$ diabetes $+0.052 \times$ age $+1.005 \times$ PSAD -9.119 . ROC curves showed the formula's threshold was 0.3543 . The composite formula had an excellent capacity to detect PCa with the area under the curve (AUC) of 0.91. In addition, the composite formula also achieved significantly better sensitivity, specificity, and diagnostic accuracy than PSA, PSA density (PSAD), and PI-RADS v2 score alone. Conclusions: Our predictive formula predicted performance better than PSA, PSAD, and PI-RADS v2 score. It can thus contribute to the diagnosis of PCa and be used as an indicator for prostate biopsy, thereby reducing unnecessary biopsy.

Keywords: Prostate cancer (PCa); prediction model; prostate biopsy; multiparametric magnetic resonance imaging prostate imaging-reporting and data system v2 (mpMRI PI-RADS v2); diabetes

Submitted Sep 02, 2019. Accepted for publication Dec 09, 2019.

doi: $10.21037 /$ tau.2019.12.42

View this article at: http://dx.doi.org/10.21037/tau.2019.12.42 


\section{Introduction}

Prostate cancer $(\mathrm{PCa})$ is the most common malignancy of the male reproductive system. 2017, PCa alone accounts for approximately 1 in 5 new cases and $8 \%$ of all cancer deaths in America (1), and is overall a significant threat to the longterm health of men. Therefore, an early diagnosis is crucial for the clinical treatment and prognosis of PCa. To improve the accuracy of discriminating $\mathrm{PCa}$, meaningful progress has been made in characterizing and developing methods, imaging techniques, and new biomarkers. Prostate-specific antigen (PSA) is the most common and cheapest screening method. However, there are many factors that have an influence on the PSA level, including age, prostate volume $(\mathrm{PV})$, prostatitis, and digital rectal examination (DRE). As it merely relies on PSA and DRE, the diagnosis value's sensitivity and specificity for early diagnosis of $\mathrm{PCa}$ are not ideal; especially when the PSA is $4-10 \mathrm{ng} / \mathrm{mL}$, the detection rate is only about $25 \%$ (2). Furthermore, in recent decades, many new markers have been found, including prostate cancer antigen 3 (PCA3) (3), prostate specific membrane antigen (PMSA) (4), PSA precursors, PSA precursor protein [proenzyme of prostate-specific antigen (proPSA)] and other indicators, all which may have more clinical significance and application prospects than PSA (5).

In addition, the growing availability of prostate magnetic resonance imaging (MRI) tools with their different functional imaging modalities and increased standardization has enlarged the role of MRI in detecting, localizing, and staging PCa. Most hospitals currently use multiparametric MRI (mpMRI) which consists of four main parameters: dynamic contrast-enhanced (DCE) imaging, T2-weighted (T2W) imaging, diffusion-weighted imaging (DWI), and MRI spectroscopy (MRS). The sensitivity of mpMRI for the diagnosis of $\mathrm{PCa}$ is $85 \%$, and the specificity is $71 \%$ (6). Recent studies have shown the potential value of using pre-biopsy mpMRI to improve the detection and characterization of clinically significant PCa. Prebiopsy mpMRI has been shown to increase the detection rate remarkably (7). Another study showed that a quarter of men had normal mpMRI and could potentially avoid an unnecessary biopsy if mpMRI was performed first (8). Prostate imaging reporting and data system (PI-RADS) v2 has been gradually incorporated into the diagnosis of $\mathrm{PCa}$. A recent study using PI-RADS to predict prostate biopsy positive rates showed significantly improved predictive efficiency (9).

According to Weinberg's theory (10), inflammation and metabolism are typically deleterious in terms of tumor emergency. More recently, an elevated neutrophillymphocyte ratio (NLR) has been put forward as an independent marker for PCa at the early and advanced stages (11). Metabolic syndrome (MS) is a group of syndromes that includes obesity, insulin resistance, dyslipidemia, hypertension, diabetes, etc. A study found that MS has a particular relationship with the onset of $\mathrm{PCa}$ (12). Some studies have examined the relationship between diabetes mellitus $(\mathrm{DM})$ and $\mathrm{PCa}$, demonstrating a reduced risk of PCa among men with DM (13); in contrast, other research indicates that patients with $\mathrm{PCa}$ and $\mathrm{DM}$ have worse overall survival (14).

Nowadays, a prostate needle biopsy is the gold standard choice for the diagnosis of PCa. There are certain limitations, even though ultrasound-guided systematic prostate biopsy misses $21 \%$ to $28 \%$ of $\mathrm{PCa}$ (15). Clinically, the indications for prostate biopsy are sometimes too broad. Additionally, a prostate biopsy is an invasive procedure with complications such as urinary retention, bleeding, and infection (16). Therefore, we urgently need a new scoring system, which not only can reduce the false positive rate, but also be more practical and accurate. Our study aimed to examine multiple predictors including age, weight, PSA, $\mathrm{PV}$, and MRI, for the individualized prediction of PCa. The diagnostic value of diabetes and NLR for PCa was also explored.

\section{Methods}

\section{Patients}

This retrospective analysis included data from 237 patients who underwent transrectal ultrasound (TRUS)-guided prostate biopsy at the Department of Urology at the First Affiliated Hospital of the University of Science and Technology of China between July 2017 and March 2018. The inclusion criteria of the patients were as follows: (I) a prostate nodule as identified by DRE, or the suspicion of PCa from imaging examination (B-ultrasound or MRI); (II) PSA 4-10 ng/mL, f/t PSA <0.16; (III) PSA > 10 ng/mL; (IV) complete clinical information. The exclusion criteria were as follows: (I) abnormal coagulation function; (II) abnormal white blood cell number in blood examination; abnormal urine routine indicating urinary system infection or acute prostatitis; (III) serious cardiopulmonary diseases; (IV) severe internal and external hemorrhoids and perianal or rectal lesions; (V) incomplete clinical information. 
All patients enrolled had undergone PI with mpMRI (3.0T Magnetom Trio MR, Siemens) before the biopsy. All patients' laboratory data were obtained within 1 week before the biopsy. Every patient enrolled had a PSA assessment, routine blood test, biochemical examination, and DRE. In addition, the following information was gathered: age, gender, body mass index (BMI), PV (measured by mpMRI), biopsy Gleason sum score (BGSS), PI-RADS v2 score, hypertension, and DM history. All laboratory and imaging examinations were carried out in the hospital, with each being conducted by a specialized professional doctor, and with pathological specimens being diagnosed by two experienced pathologists.

Prostate biopsy was guided by a TRUS biplane imaging scan (Flex Focus 800, BK Medical) with the help of mpMRI cognitive fusion. Typically, the patients underwent systematic $12+\mathrm{X}$-cores biopsy, which was based on 12 -point systematic biopsy, with needles being applied to the suspicious lesions (the target was defined as $\mathrm{X}$ ). The biopsy instruments used were the automatic biopsy gun and one-time 18G puncture biopsy needles (MCA18/20, GALLINI S.R.L.).

The NLR was calculated as the absolute neutrophil count divided by the absolute count of leukocytes. PSA density (PSAD) refers to the ratio of serum PSA concentration to PV. Diabetes and hypertension in patients were both diagnosed by endocrinologists and cardiovascular specialists. According to the probability of PCa, PI-RADS v2 scores represented the following: PI-RADS 1: very low, clinically significant cancer is highly unlikely to be present; PI-RADS 2: low, clinically significant cancer is unlikely to be present; PI-RADS 3: intermediate, the presence of clinically significant cancer is equivocal; PI-RADS 4: high, clinically significant cancer is likely to be present; PI-RADS 5: very high, clinically significant cancer is highly likely to be present (17).

\section{Statistical analyses}

The data were expressed as number (percentage), range, and median [interquartile range (IQR)]. All statistical analyses involved the use of SPSS v.22.0 (SPSS Inc., Chicago, IL, USA). $\mathrm{P}<0.05$ was considered statistically significant. Univariate and multivariate logistic analysis was used to screen factors to select the statistically significant factors, and the association between these predictors and biopsy results was then tested. We examined the significant factors in logistic regression analysis, and thereafter, the significant factors' weight indexes were included in the screening formulas. Receiver operating characteristic (ROC) curves were used to assess the discriminative ability of the variables and formulas. The maximum sensitivity and specificity of the formulas were used to determine the best threshold, while $95 \%$ confidence intervals $(95 \% \mathrm{CI})$ of the odds ratio $(\mathrm{OR})$ were used as a measure to assess relative risk.

\section{Results}

\section{The demographic characteristics of patients}

Overall, a total of 237 patients were included in this study. The descriptive parameters of the study cohort are shown in Table 1. The total positive number (rate) of needle biopsy was 92 (38.82\%). The mean (range) age, BMI, PV, and PSA, of the population was 68.23 (range, 26.00-87.00) years, 22.21 (range, $15.67-31.25$ ) kg/m², 62.03 (range, 10.91169.86 ) $\mathrm{mL}$, and 50.59 (range, $0.31-1,649.69) \mathrm{ng} / \mathrm{mL}$, respectively. The number (\%) of the BGSSs $\geq 6$ was 92 (38.82\%). The number (\%) of mpMRI PI-RADS v2 scores $>3$ was 118 (49.79\%). The mean neutrophil count, lymphocyte count, and NLR were $3.78 \times 10^{9} / \mathrm{L}, 1.76 \times 10^{9} / \mathrm{L}$, and 2.39 , respectively. Thirty-four $(14.35 \%)$ of the patients had diabetes.

\section{The formula and threshold}

Univariate logistic regression tests showed that age, PSAD, $\mathrm{DM}$, and mpMRI PI-RADS v2 scores were independent factors of PCa (Table 2) with ORs of 1.061, 7.557, 24.828, and 5.638, respectively. The OR of mpMRI PI-RADS v2 score was the largest, and it was an extremely robust risk factor for PCa. Multivariate logistic regression analyzed the age, PSAD, diabetes history, and mpMRI PI-RADS v2 score, along with the weighting indexes of these factors for PCa prediction (Table 3). The men with PCa identified by biopsy tended to be older, suffer diabetes, and have higher PSAD levels and mpMRI PI-RADS v2 scores.

Based on the weighting indexes of multivariate logistic regression analysis, we derived the following formula: $\mathrm{Y}$ $=2.599 \times \mathrm{mpMRI}$ PI-RADS v2 score $+1.766 \times$ diabetes + $0.052 \times$ age $+1.005 \times$ PSAD -9.119

ROC curves indicated that 0.3543 was the threshold of the formula, which constituted the formula's prediction sensitivity and specificity maximum. When applied to an individual, the $\mathrm{Y}$ value is calculated in combination with the patient's age, PSAD, mpMRI PI-RADS v2 score, and 
Table 1 Patients' characteristics and descriptive statistics

\begin{tabular}{|c|c|}
\hline Characteristics & Statistics \\
\hline Total, n (\%) & $237(100.00)$ \\
\hline \multicolumn{2}{|l|}{ Age, years } \\
\hline Mean (median) & $68.23(69.00)$ \\
\hline Range & $26.00-87.00$ \\
\hline $10^{\text {th }}-90^{\text {th }}$ percentile & $57.00-78.00$ \\
\hline \multicolumn{2}{|l|}{$\mathrm{BMI}, \mathrm{kg} / \mathrm{m}^{2}$} \\
\hline Mean (median) & $22.21(21.97)$ \\
\hline Range & $15.67-31.25$ \\
\hline $10^{\text {th }}-90^{\text {th }}$ percentile & $18.37-28.82$ \\
\hline \multicolumn{2}{|l|}{$\mathrm{PV}, \mathrm{mL}$} \\
\hline Mean (median) & $62.03(55.31)$ \\
\hline Range & $10.91-169.86$ \\
\hline $10^{\text {th }}-90^{\text {th }}$ percentile & $28.45-103.16$ \\
\hline \multicolumn{2}{|l|}{$\mathrm{PSA}, \mathrm{ng} / \mathrm{mL}$} \\
\hline Mean (median) & $50.59(14.00)$ \\
\hline Range & $0.31-1,649.69$ \\
\hline $10^{\text {th }}-90^{\text {th }}$ percentile & $7.36-100.00$ \\
\hline \multicolumn{2}{|c|}{ PI-RADS v2 score, n (\%) } \\
\hline$\leq 2$ & 55 (23.21) \\
\hline 3 & $64(27.00)$ \\
\hline$>3$ & $118(49.79)$ \\
\hline \multicolumn{2}{|l|}{ BGSS, n (\%) } \\
\hline$\leq 6$ & $145(61.18)$ \\
\hline 6 & $17(7.17)$ \\
\hline 7 & $25(10.55)$ \\
\hline$\geq 8$ & $50(21.10)$ \\
\hline \multicolumn{2}{|l|}{ Hypertension, n (\%) } \\
\hline $\mathrm{Y}$ & $82(34.60)$ \\
\hline $\mathrm{N}$ & $155(65.40)$ \\
\hline \multicolumn{2}{|l|}{ DM, n (\%) } \\
\hline Y & 34 (14.35) \\
\hline $\mathrm{N}$ & $203(85.65)$ \\
\hline
\end{tabular}

Table 1 (continued)
Table 1 (continued)

\begin{tabular}{lc}
\hline Characteristics & Statistics \\
\hline Neutrophil count, $10^{9} / \mathrm{L}$ & $3.78(3.69)$ \\
Mean (median) & $1.28-10.14$ \\
Range & $2.42-5.31$ \\
$10^{\text {th }}-90^{\text {th }}$ percentile & \\
Lymphocyte count, $10^{9} / \mathrm{L}$ & $1.76(1.68)$ \\
Mean (median) & $0.50-3.66$ \\
Range & $1.11-2.61$ \\
$10^{\text {th }}-90^{\text {th }}$ percentile & \\
NLR & $2.39(2.16)$ \\
Mean (median) & $0.69-11.52$ \\
Range & $1.30-3.72$ \\
$10^{\text {th }}-90^{\text {th }}$ percentile
\end{tabular}

BMI, body mass index; PV, prostate volume; PSA, prostatespecific antigen; PI-RADS, prostate imaging-reporting and data system; BGSS, biopsy Gleason sum score; Y, yes; N, no; DM, diabetes mellitus; NLR, neutrophil-to-lymphocyte ratio.

Table 2 Univariate logistic regression analyses for predicting prostate cancer

\begin{tabular}{lccc}
\hline Indicator & OR & $95 \% \mathrm{Cl}$ & $\mathrm{P}$ \\
\hline Age & 1.061 & $1.025-1.098$ & 0.001 \\
PSAD & 7.557 & $3.841-14.866$ & 0.000 \\
PI-RADS v2 score & 24.828 & $11.647-52.926$ & 0.000 \\
DM & 5.638 & $2.493-12.753$ & 0.000 \\
\hline
\end{tabular}

OR, odds ratio; $95 \% \mathrm{Cl}, 95 \%$ confidence intervals; PSAD, prostate-specific antigen density; PI-RADS, prostate imagingreporting and data system; DM, diabetes mellitus.

presence of diabetes. When the $\mathrm{Y}$ value is $>0.3543$, prostate biopsy is indicated.

\section{Comparisons of different screening methods}

The ROC curve was used to compare the formula's predictive value for PCa with patients' age, PSAD, and mpMRI PI-RADS v2 score (Figure 1). The thresholds of 
Table 3 The result of multivariate logistic regression for indicators

\begin{tabular}{lcccccc}
\hline Indicator & $\mathrm{B}$ & $\mathrm{S}$ & WALS value & OR & $95 \% \mathrm{Cl}$ \\
\hline Age & 0.052 & 0.024 & 4.658 & 1.053 & $1.005-1.104$ & 0.031 \\
PSAD & 1.005 & 0.328 & 9.287 & 2.731 & $1.436-5.194$ \\
PI-RADS v2 score & 2.599 & 0.435 & 35.643 & 13.455 & $5.732-31.585$ \\
DM & 1.766 & 0.569 & 9.615 & 5.845 & $1.915-17.844$ & 0.002 \\
Constant & -9.119 & 1.861 & 24.002 & 0.000 & -0.002 & 0.000 \\
\hline
\end{tabular}

OR, odds ratio; 95\% Cl, 95\% confidence intervals; PSAD, prostate-specific antigen density; PI-RADS, prostate imaging-reporting and data system; DM, diabetes mellitus.
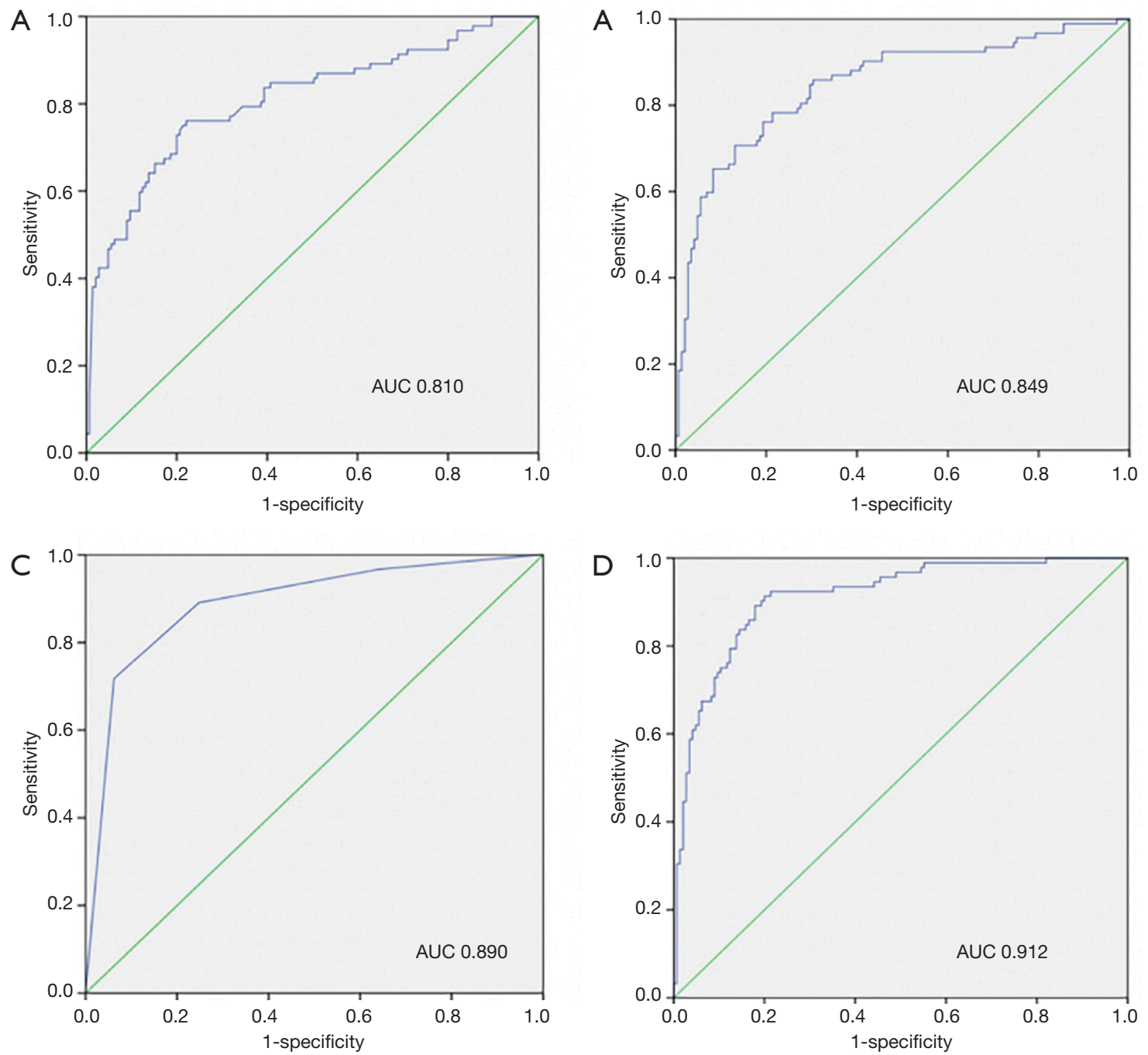

Figure 1 ROC curves for PSA, PSAD, mpMRI PI-RADS v2 score, and our formula model. (A) PSA, AUC: 0.810; (B) PSAD, AUC: 0.849; (C) mpMRI PI-RADS v2 score, AUC: 0.890; (D) our formula model, AUC: 0.912. ROC, receiver operating characteristic; PSA, prostatespecific antigen; PSAD, PSA density; mpMRI, multiparametric magnetic resonance imaging; PI-RADS, prostate imaging-reporting and data system; AUC, area under the curve. 
PSA and PSAD were $10 \mathrm{ng} / \mathrm{mL}$ and 0.485 respectively, with the area under the curve (AUC) of these screening methods ranging from 0.810 to 0.912 . The detective formula could best detect PCa with an AUC of 0.912, compared with AUCs of 0.810, 0.849, and 0.890 for PSA level, PSAD, and mpMRI PI-RADS v2 score, respectively (Figure 2).

Moreover, we used sensitivity, specificity, positive predictive value (PPV), negative predictive value (NPV), overall diagnostic accuracy (ODA), positive likelihood

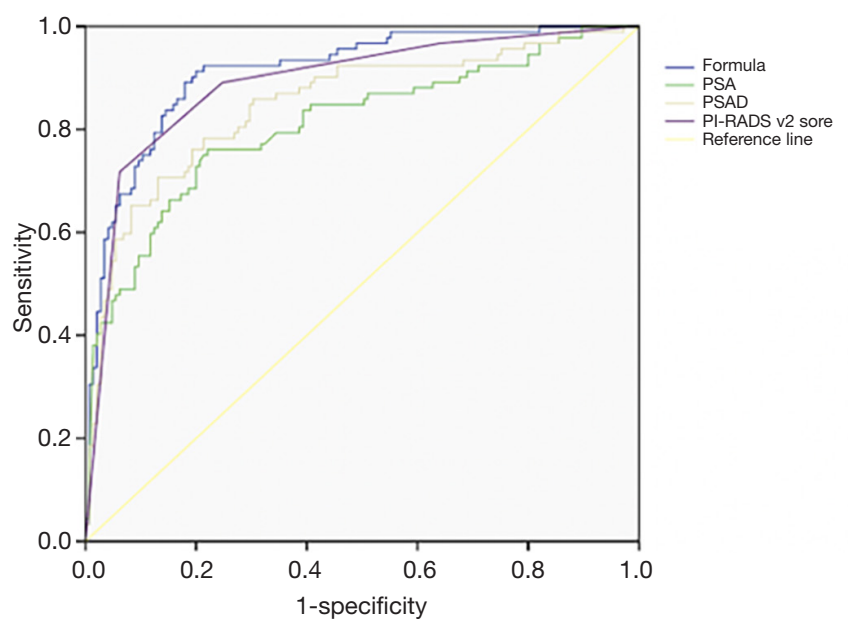

Figure 2 Comparison of ROC curves among PSA, PSAD, mpMRI PI-RADS v2 score, and our formula model. The formula model demonstrated the best capacity for the detection of $\mathrm{PCa}$ with an AUC of 0.912. ROC, receiver operating characteristic; PSA, prostate-specific antigen; PSAD, PSA density; mpMRI, multiparametric magnetic resonance imaging; PI-RADS, prostate imaging-reporting and data system; PCa, prostate cancer; AUC, area under the curve. ratio (+LR), and negative likelihood ratio (-LR) to analyze and compare the diagnostic value and clinical application value of the formula and other methods (Table 4). PSA had the lowest ODA (56.54\%). The detective formulas achieved optimum sensitivity (91.30\%), NPV (93.55\%), and ODA (84.39\%) over those of PSA and PSAD alone. The specificity $(80.00 \%)$ and PPV $(74.34 \%)$ of the detective formula were lower than PSAD. +LR of the formula was larger than PSA (4.57/1.20), and the formula achieved the minimum -LR.

\section{Discussion}

We investigated the serological tumor markers, imaging features, and history of tumor-related metabolic diseases in 237 patients with suspected PCa. We demonstrated that decreased age, PSAD, mpMRI, and diabetes were independent and adverse predictors in the logistic analysis. The incidence of $\mathrm{PCa}$ is increasing annually with an aging population. The early screening markers for PCa are thus extremely important, with the current markers being predominantly serum PSA. Most urologists judge whether to perform prostate biopsy based on these PSA results. Nevertheless, PSA is susceptible to many negative factors, and its prediction for $\mathrm{PCa}$ is not only insensitive but also too generalized. To better predict $\mathrm{PCa}$, scholars have proposed different PCa prediction models $(18,19)$, with poor predictive performance. In addition, researchers have created PSAD, which is based on PSA and PV. PSAD attenuates the effect of PV on PSA, and thus PSAD is considered to be superior to PSA in identifying PCa, especially when the PSA concentration is in the gray area (4-10 ng/mL) (20).

Table 4 Comparison of performance of PSA, PSAD and detection formula

\begin{tabular}{lccc}
\hline Indicator & PSA (\%) & PSAD (\%) & Formula (\%) \\
\hline Sensitivity & $82 / 92(89.13)$ & $65 / 92(70.65)$ & $84 / 92(91.30)$ \\
Specificity & $52 / 145(35.86)$ & $126 / 145(86.90)$ & $116 / 145(80.00)$ \\
PPV & $82 / 175(46.86)$ & $65 / 84(77.38)$ & $84 / 113(74.34)$ \\
NPV & $52 / 62(83.87)$ & $126 / 153(82.35)$ & $116 / 124(93.55)$ \\
+LR & 1.20 & 5.39 & 4.57 \\
-LR & 0.42 & 0.34 & 0.11 \\
ODA & $(56.54)$ & $(80.59)$ & $(84.39)$ \\
\hline
\end{tabular}

PSA, prostate-specific antigen; PSAD, PSA density; PPV, positive predictive value; NPV, negative predictive value; +LR, positive likelihood ratio; -LR, negative likelihood ratio; ODA, overall diagnostic accuracy. 
mpMRI is one of the most important imaging tests for the diagnosis of PCa. It is not only able to detect an early-stage tumor but it can also predict the Gleason score of $\mathrm{PCa}$ (21). Pre-biopsy mpMRI is more accurate in assessing the extent and risk of $\mathrm{PCa}$, and the excellent diagnostic accuracy of mpMRI PI-RADS v2 score has been conclusively proven (22). When it and other indicators were combined, the judgment threshold was even higher. Many studies showed that when PI-RADS v2 score was combined with PSAD, the screening efficiency of biopsy improved greatly, resulting in a near $50 \%$ reduction in unnecessary biopsies $(23,24)$. In the present study, the PI-RADS v2 score has the highest OR value in multivariate logistic regression analysis, showing that the higher the value, the higher the risk of PCa. In our data, 118 (49.79\%) patients' PI-RADS v2 score was $>3$, and, according to the guidelines, this indicates that the possibility of $\mathrm{PCa}$ is high. When the patient is in a low-risk group (PI-RADS v2 score $\leq 3$ ), appropriate decisions should be made to avoid unnecessary needle biopsy.

We also observed other statistically significant phenomena; DM was an independent risk factor for $\mathrm{PCa}$; that is, diabetic patients are more prone to $\mathrm{PCa}$. Nevertheless, the influence of DM on the pathogenesis of $\mathrm{PCa}$ is still controversial, and its mechanisms remain unclear. It may be related to factors such as blood glucose control level, insulin resistance, and hyperinsulinemia in DM patients (25). In addition, some researchers have found that hypoglycemic drugs also have an impact on PCa. A study in Taiwan showed that metformin reduced the risk of $\mathrm{PCa}$ in patients with type $2 \mathrm{DM}$. Another study found that metformin reduced $\mathrm{PCa}$ patients' biochemical recurrence rate, risk of distant metastasis, and tumor-specific mortality $(26,27)$. Based on this, we recommend that diabetic patients should actively control blood sugar to ensure it is within a reasonable range when applying rational hypoglycemic drugs.

Unexpectedly, our findings showed that NLR was not an independent risk factor of PCa. Since Virchow first addressed the link between cancer and inflammation in the 19th century, contemporary research has generally recognized that inflammation plays a vital role in carcinogenesis (28). Researchers have revealed that inflammation promotes $\mathrm{PCa}$ (29), while Cainozoic evidence has shown that NLR is a valuable prognostic factor in PCa. High NLR in pretreatment was a poor prognostic factor for survival in patients. Most studies have found that NLR is an important predictor of PCa prognosis (overall survival, progression-free survival, recurrence-free survival, etc.) (11), but the diagnostic value of PCa may need further study.

Furthermore, we created a predictive formula based on the patient's age, PSAD, mpMRI PI-RADS v2 score, and DM. We tested and compared the predictive value of the formula and other screening methods. The formula had a higher screening evaluation index. The order of AUC values was PSA $<$ PSAD < MRI PI-RADS v2 score < our formula. The AUC of the ROC curve of the formula was the largest, showing the diagnostic value of the formula is the best. In addition, the detective formulas achieved optimum sensitivity, NPV, and overall accuracy, but the specificity and PPV were lower. High sensitivity means more patients are screened out, leading to fewer patients missing out on chances for early treatment, which can result in a poor prognosis. The specificity of the formula was approximately 3 times that of PSA, and its application could significantly reduce unnecessary prostate biopsy and prevent harm to patients. Because PPV and NPV vary with prevalence, they cannot be used as an index to evaluate the value of diagnostic tests. The +LR of the formula was much greater than that of PSA, while the -LR was the smallest. This means that the formula can screen out more positive patients while eliminating unnecessary puncture patients, but they still should be closely observed.

Finally, some limitations to our study should be addressed. First, the cohort of the study was not an optimum screening population but a hospitalized population in a single hospital. There was undoubtedly a selection offset. Second, owing to the retrospective nature and the lack of external validation, the predictive significance of the formula remains to be prospectively studied in future populations and larger cohorts. Third, our study did not include other indicators of MS, such as triglycerides, cholesterol, etc. Further research should fully estimate the predictive value of MS. Last, the men with PCa were diagnosed by biopsy using pathological specimens obtained by TRUS-guided biopsy, and thus there was a lack of comparison to other biopsy pathways. A difference in operation might have caused a missed diagnosis, which could have led to bias in our research.

Overall, our findings suggest that age, PSAD, mpMRI PI-RADS v2 score, and DM are independent risk factors of $\mathrm{PCa}$, as they showed superior performance in detecting PCa. These factors could provide clinicians with better screening methods to predict PCa before biopsy. In addition, DM was closely associated with $\mathrm{PCa}$, and thus diabetic patients are more likely to develop PCa. 


\section{Acknowledgments}

Funding: This study was supported by the National Natural Science Foundation of China (81702540) and the Anhui Natural Science Foundation (1708085QH202).

\section{Footnote}

Conflicts of Interest: All authors have completed the ICMJE uniform disclosure form (available at http://dx.doi. org/10.21037/tau.2019.12.42). The authors have no conflicts of interest to declare.

Ethical Statement: The authors are accountable for all aspects of the work in ensuring that questions related to the accuracy or integrity of any part of the work are appropriately investigated and resolved. All patients included in the study had signed a written informed consent and agreed to store their individual case information in a hospital database for research. This study was approved by the ethics committee of Anhui Medical University (No. 20170179).

Open Access Statement: This is an Open Access article distributed in accordance with the Creative Commons Attribution-NonCommercial-NoDerivs 4.0 International License (CC BY-NC-ND 4.0), which permits the noncommercial replication and distribution of the article with the strict proviso that no changes or edits are made and the original work is properly cited (including links to both the formal publication through the relevant DOI and the license). See: https://creativecommons.org/licenses/by-ncnd/4.0\%.

\section{References}

1. Siegel RL, Miller KD, Jemal A. Cancer statistics, 2017. CA Cancer J Clin 2017;67:7-30.

2. Stamey TA, Johnstone IM, McNeal JE, et al. Preoperative serum prostate specific antigen levels between 2 and 22 $\mathrm{ng} . / \mathrm{ml}$. correlate poorly with post-radical prostatectomy cancer morphology: prostate specific antigen cure rates appear constant between 2 and $9 \mathrm{ng} . / \mathrm{ml}$. J Urol 2002;167:103-11.

3. Lazzeri M, Haese A, de la Taille A, et al. Serum isoform [-2]proPSA derivatives significantly improve prediction of prostate cancer at initial biopsy in a total PSA range of 2-10 ng/ml: a multicentric European study. Eur Urol
2013;63:986-94.

4. Ben Jemaa A, Bouraoui Y, Sallami S, et al. Cellular distribution and heterogeneity of Psa and Psma expression in normal, hyperplasia and human prostate cancer. Tunis Med 2013;91:458-63.

5. Rönnau CG, Verhaegh GW, Luna-Velez MV, et al. Noncoding RNAs as novel biomarkers in prostate cancer. Biomed Res Int 2014;2014:591703.

6. Zhang L, Tang M, Chen S, et al. A meta-analysis of use of prostate imaging reporting and data system version 2 (PI-RADS V2) with multiparametric MR imaging for the detection of prostate cancer. Eur Radiol 2017;27:5204-14.

7. Kasivisvanathan V, Rannikko AS, Borghi M, et al. MRItargeted or standard biopsy for prostate-cancer diagnosis. N Engl J Med 2018;378:1767-77.

8. Ahmed HU, El-Shater Bosaily A, Brown LC, et al. Diagnostic accuracy of multi-parametric MRI and TRUS biopsy in prostate cancer (PROMIS): a paired validating confirmatory study. Lancet 2017;389:815-22.

9. Grey AD, Chana MS, Popert R, et al. Diagnostic accuracy of magnetic resonance imaging (MRI) prostate imaging reporting and data system (PI-RADS) scoring in a transperineal prostate biopsy setting. BJU Int 2015;115:728-35.

10. Hanahan D, Weinberg RA. Hallmarks of cancer: the next generation. Cell 2011;144:646-74.

11. Minardi D, Scartozzi M, Montesi L, et al. Neutrophil-tolymphocyte ratio may be associated with the outcome in patients with prostate cancer. Springerplus 2015;4:255.

12. Liu J, Zhang Y, Du X, et al. Metabolic syndrome components were related to the risks of prostate cancer. Int J Clin Exp Med 2016;9:18500-6.

13. Miller EA, Pinsky PF, Pierre-Victor D. The relationship between diabetes, prostate-specific antigen screening tests, and prostate cancer. Cancer Causes Control 2018;29:907-14.

14. Karlin NJ, Amin SB, Verona PM, et al. Co-existing prostate cancer and diabetes mellitus: implications for patient outcomes and care. Endocr Pract 2017;23:816-21.

15. Bjurlin MA, Carter HB, Schellhammer P, et al. Optimization of initial prostate biopsy in clinical practice: sampling, labeling and specimen processing. J Urol 2013;189:2039-46.

16. Xue J, Qin Z, Cai H, et al. Comparison between transrectal and transperineal prostate biopsy for detection of prostate cancer: a meta-analysis and trial sequential analysis. Oncotarget 2017;8:23322-36.

17. Weinreb JC, Barentsz JO, Choyke PL, et al. PI-RADS 
prostate imaging - reporting and data system: 2015, version 2. Eur Urol 2016;69:16-40.

18. Tang P, Chen H, Uhlman M, et al. A nomogram based on age, prostate-specific antigen level, prostate volume and digital rectal examination for predicting risk of prostate cancer. Asian J Androl 2013;15:129-33.

19. Blute ML Jr, Abel EJ, Downs TM, et al. Addressing the need for repeat prostate biopsy: new technology and approaches. Nat Rev Urol 2015;12:435-44.

20. Tang P, Du W, Xie K, et al. Transition zone PSA density improves the prostate cancer detection rate both in PSA 4.0-10.0 and 10.1-20.0 ng/ml in Chinese men. Urol Oncol 2013;31:744-8.

21. Wang X, Tu N, Qin T, et al. Diffusion kurtosis imaging combined with DWI at 3-T MRI for detection and assessment of aggressiveness of prostate cancer. AJR Am J Roentgenol 2018;211:797-804.

22. Muller BG, Shih JH, Sankineni S, et al. Prostate cancer: interobserver agreement and accuracy with the revised prostate imaging reporting and data system at multiparametric MR imaging. Radiology 2015;277:741-50.

23. Alberts A, Roobol M, Drost FJ, et al. PSA-density based patient selection for MRI-targeted prostate biopsy could reduce unnecessary biopsy procedures in men on active surveillance for low-grade prostate cancer. European

Cite this article as: Tao T, Shen D, Yuan L, Zeng A, Xia K, Li B, Ge Q, Xiao J. Establishing a novel prediction model for improving the positive rate of prostate biopsy. Transl Androl Urol 2020;9(2):574-582. doi: 10.21037/tau.2019.12.42
Urology Supplements 2017;16:e856-8.

24. Washino S, Okochi T, Saito K, et al. Combination of prostate imaging reporting and data system (PI-RADS) score and prostate-specific antigen (PSA) density predicts biopsy outcome in prostate biopsy naïve patients. BJU Int 2017;119:225-33.

25. Yin M, Zhou J, Gorak EJ, et al. Metformin is associated with survival benefit in cancer patients with concurrent type 2 diabetes: a systematic review and meta-analysis. Oncologist 2013;18:1248-55.

26. Tseng CH. Metformin significantly reduces incident prostate cancer risk in Taiwanese men with type 2 diabetes mellitus. Eur J Cancer 2014;50:2831-7.

27. Spratt DE, Zhang C, Zumsteg ZS, et al. Metformin and prostate cancer: reduced development of castrationresistant disease and prostate cancer mortality. Eur Urol 2013;63:709-16.

28. Balkwill F, Mantovani A. Inflammation and cancer: back to Virchow? Lancet 2001;357:539-45.

29. Lanciotti M, Masieri L, Raspollini MR, et al. The role of M1 and M2 macrophages in prostate cancer in relation to extracapsular tumor extension and biochemical recurrence after radical prostatectomy. Biomed Res Int 2014;2014:486798. 